commonest ADE found. Age under 4, length of stay in PICU and number of drugs used are risk factors to $\mathrm{ADE}$ development.

\section{USE OF STATINS IN CHILDREN}

doi:10.1136/archdischild-2012-302724.0088

EE Moreno Medinilla, L Ferreras Antolín, F Girón Fernández-Creueht, J Blasco Alonso, J Serrano Nieto, V Navas López, A Jurado Ortíz, C Sierra Salinas. Hospital Marterno Infantil Carlos Haya, Málaga, Spain

Introduction Referring to the use of statins for hyperlipemia in children, it is not yet clearly defined who must be treated (recent recommendations focused on older than 8 years) and also the frequency of short and long-term toxicity.

Methods Retrospective, descriptive study of patients, 10 year-old or younger, under statin treatment, followed in our Unit. Epidemiology, treatment and side-effects data were analized.

Results Twelve children met inclusion criteria; 7/12 female and $11 / 12$ caucasian. Main diagnosis was familiar hypercholesterolemia (10/12); 1/12 hypertriglyceridemia and 1/12 nefrotic syndrome. Positive family history of cardiovascular events: 5/12 (no death among first degree relatives). Mean age at diagnosis was 5.3 years. All of them were asymptomatic. $8 / 12$ had been treated previously (7/12 resins, 1/12 cholesterol absorption inhibitor). Statin treatment starting age was 8.6 years, $4 / 12$ younger than 8 year-old (minimum age: 5.2 years). Atorvastatin used in 10/12, lovastatin 1/12, simvastatin $1 / 12$; initial dose was always $10 \mathrm{mg} /$ day. The average levels before treatment were: LDL $235 \mathrm{mg} / \mathrm{dL}$, total cholesterol $324.7 \mathrm{mg} / \mathrm{dL}$. In one 10 year-old patient (40 mg/day lovastatin) muscular pain was reported with a CPK increase; side-effects stopped once the statin dose was reduced to $20 \mathrm{mg} /$ day. Besides, no adverse effect was reported. The highest dose achieved was $40 \mathrm{mg} /$ day. Patients follow-up varies from 9 months to 4 years.

\section{Conclusions}

- It remains unclear whether statins could be started before 8 year-old. From our experience, no adverse effects were found in that group.

- Dose had to be increased to $20-40 \mathrm{mg} /$ day in order to achieve objectives.

\section{CLINICAL JUDGEMENT AND PAIN ASSESSMENT IN CRITICALLY ILLCHILDREN}

doi:10.1136/archdischild-2012-302724.0089

${ }^{1,2} \mathrm{AL}$ Thevoz, ${ }^{1,3} \mathrm{~A}$ Gerber, ${ }^{1,3} \mathrm{AS}$ Ramelet. '/nstitute of Higher Education and Nursing Research, University of Lausanne; ${ }^{2}$ Haute Ecole de la Santé La Source; ${ }^{3}$ Haute Ecole de Santé Vaud, University of Applied Sciences Western Switzerland, Lausanne, Switzerland

Background and aims Despite the various pain assessment tools available to date, pain assessment in critically ill children remains challenging for nurses. Clinical judgement of pain depends on different factors that have yet to be described in real-life settings. The aim of this observational study was to describe expert nurses' clinical judgment when assessing pain in critically ill children.

Methods Following ethics approval and participants' consent, a convenience sample of expert nurses working in a tertiary referral paediatric intensive care unit (PICU) in Western Switzerland participated in the study. Data were collected using the think-aloud method, combined with non-participant observation and semistructured interviews. Data were analysed using deductive content analyses based on the O'Neil's decision-making model. Categories

Results The ten nurse participants had an average of 12.9 years of nursing experience. Seven intubated and ventilated patients were observed. Four had cardiac surgery, one diaphragmatic hernia, one tracheal reconstruction, and one respiratory syndrome. Results show that pre-encountered data and knowing the patient are important factors. Expert nurses mobilise their knowledge to discriminate between pain-related agitation and agitation caused by other factors by generating hypothesis. They perform analgesic tests to confirm or refute pain. Counter-balancing the benefits and adverse effects of analgesia and sedation is also part of their clinical judgement when making decision about pain management.

Conclusions The clinical context of the patient plays an important part in nurses' judgment about pain. To facilitate this difficult task, pain assessment should be combined with sedation assessment in critically ill children.

\section{OBSERVATIONAL STUDY ON PAIN AND DISTRESS IN CHINESE AND ITALIAN CHILDREN UNDERGOING VENIPUNCTURE}

doi:10.1136/archdischild-2012-302724.0090

F Festini, M Calzolai, S Bisogni. University of Florence, Florence, Italy

Few studies have examined the influence of ethnic-cultural variables on pain perception and behavioural distress in children. Not considering possible cultural variations of pain manifestation may lead to inadequate assessment and treatment of pain.

Goal To evaluate wether differences exist between Italian and nonItalian children with regards to pain perception and behavioural distress during the same invasive procedure.

Methods Cross-sectional analytical trial. A group of Italian children (group A) and a group of Chinese children (group B) aged 3 to 11 were observed during a standardized venipuncture for blood sample drawing. Pain was self-rated with a 1-10 Wong faces scale or a 1-10 numeric scale. Behavioural distress was measured with the Observational Behaviour Distress Scale (OBDS, 1-33).

Results 246 children were examined, 191 in group A and 55 in group $B$. In preschool age ( 3 to $5, n=76$ ), neither mean pain rates nor mean OBDS rates were statistically different in the two groups. On the opposite, in the $6-11$ age $(n=170)$, mean pain rate was 2.9 in group $\mathrm{A}$ and 4.8 in group $\mathrm{B}$ (Anova $\mathrm{p}=0.00001$ ) while mean OBDS rate was 6.6 in group $A$ and 2.3 in group $B i$ (Anova $p=0.0005$ ).

Discussion Our data show that duiring venipuncture Chinese children have less marked behavioural distress manifestations than their Italian peers, even though the perceived pain is higher in Chinese children.

\section{AUDIT OF PAIN, SEDATION AND WITHDRAWAL PRACTICES IN THE UK, IRELAND AND THE NETHERLANDS}

doi:10.1136/archdischild-2012-302724.0091

'J Harris, ${ }^{2} \mathrm{~L}$ Bakker, ${ }^{3} \mathrm{~L}$ Tume, ${ }^{4} \mathrm{E}$ Ista, Paediatric Intensive Care Society - Nursing Study Group for Pain, SedationDrug Withdrawal. 'Paediatric Intensive Care, Guy's \& St Thomas' NHS Foundation Trust, London, UK; 'Paediatric Intensive Care, Radboud University Nijmegen Medical Centre, Nijmegen, The Netherlands; ${ }^{3 P a e d i a t r i c ~ I n t e n s i v e ~}$ Care, Alder Hey Childrens' Hospital, Liverpool, UK; 'Erasmus MC - Sophia Childrens Hospital, Rotterdam, The Netherlands

Background and aims Assessment and management of pain, sedation and drug withdrawal in PICU's is notoriously difficult. This audit aimed to compare practices across the UK, Ireland (UK\&I) and The Netherlands (NL) with regards to pain, sedation and drug withdrawal.

Methods An electronic questionnaire was sent to all PICU's listed in the PICANET database in January 2011 and to all PICU's in The Netherlands. The questionnaire was sent to the lead nurse, lead doctor, educator or research nurse.

Results Response rate: UK\&I was 51\% (18/35) and for NL 100\% (8/8), respectively. 